\title{
Квантование проводимости в мемристивных структурах на основе поли-п-ксилилена
}

\author{
(C) Б.С. Швецов ${ }^{1,2}$, А.А. Миннеханов ${ }^{2}$, А.А. Несмелов ${ }^{2}$, М.Н. Мартышов ${ }^{1}$, В.В. Рыльков ${ }^{2,3}$, \\ B.A. Демин ${ }^{2}$, А.В. Емельянов ${ }^{2,4}$ \\ ${ }^{1}$ Московский государственный университет им. М.В. Ломоносова (физический фракультет), \\ 119991 Москва, Россия \\ ${ }^{2}$ Национальный исследовательский центр „Курчатовский институт“, \\ 123182 Москва, Россия \\ ${ }^{3}$ Фрязинский фрилиал Института радиотехники и электроники им. В.А. Котельникова Российской академии наук, \\ 141190 Фрязино, Россия \\ ${ }^{4}$ Московский фризико-технический институт, \\ 141700 Долгопрудный, Россия \\ E-mail: b.shvetsov15@physics.msu.ru
}

Поступила в Редакцию 15 апреля 2020 г.

В окончательной редакции 21 апреля 2020 г.

Принята к публикации 21 апреля 2020 г.

\begin{abstract}
Представлены результаты исследования при комнатных температурах эффекта квантования проводимости мемристивных структур на основе органического материала поли-п-ксилилена при резистивном переключении. Показаны методы измерения и проведен сравнительный анализ проявления эффекта при переключении структур в высокоомное и низкоомное состояния. Продемонстрирована возможность задания стабильных квантовых состояний проводимости в мемристивных структурах на основе поли-п-ксилилена. Показано, что некоторые из этих состояний обладают кратковременной, а некоторые долговременной стабильностью. Полученные результаты открывают новые возможности использования эффекта квантования проводимости в реализации нейроморфных систем.
\end{abstract}

Ключевые слова: мемристор, органическая электроника, поли-п-ксилилен, квантование проводимости.

DOI: 10.21883/FTP.2020.09.49831.23

\section{1. Введение}

Мемристоры (резисторы с памятью) - устройства, основанные на эффекте обратимого резистивного переключения (РП). Их главная функциональная особенность заключается в способности изменять сопротивление в зависимости от приложенного напряжения и протекающего тока, а также сохранять возникающее резистивное состояние после снятия напряжения. Явления РП между высоко- и низкоомным состояниями в структурах металл/диэлектрик/металл (МДМ) на основе оксидов были обнаружены более 50 лет назад (см., например, обзор [1]). Однако повышенный интерес к их исследованию возник сравнительно недавно, после обнаружения мемристивных свойств МДМ структур на основе нанослоев $\mathrm{TiO}_{2-x}$ [2]. Движение в оксиде кислородных вакансий под действием сильного электрического поля, обнаруженное в [2], позволяет непрерывно и обратимо изменять/сохранять электрическое сопротивление МДМ структур. Это открывает перспективы их использования для создания многоуровневой энергонезависимой памяти или элементов, моделирующих функции синапса (соединения между нейронами) при построении нейромофных вычислительных систем для решения так называемых антропоморфных задач [3-5].

За последнее десятилетие обнаружено большое количество различных объектов, обладающих эффектом
РП, которые можно классифицировать в зависимости от его механизма [6,7]. Наблюдаемые в МДМ структурах эффекты РП обычно объясняют процессами электрополевого дрейфа вакансий кислорода VO в слое диэлектрического оксида $\left(\mathrm{TiO}_{x}, \mathrm{HfO}_{x}, \mathrm{TaO}_{x}\right.$ и др.) $[1,4,6,7]$ или электромиграции катионов металлов типа $\mathrm{Cu}, \mathrm{Ag}$ из активного электрода МДМ структуры в диэлектрик (например, $\mathrm{SiO}_{2}$, органика) [8,9]. Как результат, в диэлектрическом слое образуются (разрушаются) нитевидные хорошо проводящие каналы либо металлические мостики, формирование (разрушение) которых обусловлено передвижением вакансий кислорода или катионов металла соответственно. При этом структура под действием внешнего электрического поля может обратимо переключаться между состоянием с высоким сопротивлением (HRS, или OFF состоянием) и состоянием с низким сопротивлением (LRS, или ON состоянием).

Механизм электрохимической металлизации предполагает, что катионы металла верхнего электрода под действием положительного напряжения мигрируют в слой диэлектрика и далее к нижнему электроду, где восстанавливаются и образуют так называемый металлический мостик, замыкающий верхний и нижний электроды [8-11]. При этом сопротивление мемристора, изначально находившегося в состоянии OFF, уменьшается, и структура переходит в ON состояние. При приложении к верхнему электроду отрицательного напряжения наи- 
более тонкий конец мостика разрушается, часть ионов металла возвращается обратно в электрод, и структура переключается в OFF состояние.

В этом отношении представляют интерес мемристивные элементы на основе органических материалов, так как они не уступают неорганическим мемристорам по основным характеристикам: отношению сопротивлений в высоко- и низкоомном состояниях, времени хранения состояний (стабильность), количеству циклов переключения без деградации (выносливость) [12-15]. В то же время многие органические материалы обладают рядом несомненных преимуществ: низкая стоимость и простота изготовления, биосовместимость, возможность исполнения на гибких подложках и трехмерного конструирования материала [13-16]. Одними из наиболее перспективных подобных структур являются мемристоры на основе слоев поли-п-ксилилена (также парилен, или PРX) [15] — полимера, используемого в основном в электронике для создания диэлектрических покрытий. Мемристоры на основе PPX демонстрируют хорошие характеристики: отношение сопротивлений в высоко- и низкоомном состояниях составляет $10^{2}-10^{3}$, количество циклов переключения - не менее 1000, а количество промежуточных резистивных состояний достигает 16, каждое из которых сохраняется до нескольких часов [17]. Кроме того, данные мемристивные структуры демонстрируют возможность изменения проводимости по биоподобным правилам типа STDP (сокр. от spike-timing-dependent plasticity - пластичности, зависящей от времени прихода импульсов) [5]. При этом структуры на основе РPХ могут быть созданы на гибких подложках, исследование которых показало устойчивость эффекта РП к механическим изгибам вплоть до радиусов в 7 мм [18].

Особый интерес к исследованию эффектов РП в МДМ структурах связан с обнаружением в них эффекта квантования проводимости при комнатной температуре, наблюдаемого обычно в режиме перехода структуры в OFF состояние, т.е. при уменьшении диаметра $d$ проводящих мостиков до размеров $d \leq \lambda_{\mathrm{F}}$ [19-22] $\left(\lambda_{\mathrm{F}}-\right.$ фермиевская длина волны электрона). В этих условиях проводимость структуры $G$ может быть выражена как

$$
G=n\left(2 e^{2} / h\right)=n \cdot G_{0}, \quad n=(1,2,3, \ldots),
$$

где $e-$ заряд электрона, $h-$ постоянная Планка, множитель 2 учитывает вырождение по спину, а $G_{0}=2 e^{2} / h-$ квант проводимости $\left(G_{0}=\right.$ $=77.5$ мкмСм [23]).

Данный эффект представляется интересным для физического понимания явления РП в мемристивных структурах, а кроме того, для изучения возможности реализации новых сверхплотных схем хранения информации, логически-запоминающих схем и т.д. [24]. Однако, несмотря на значительные усилия по исследованию в мемристорах эффекта квантования проводимости, ряд вопросов до сих пор остается не выясненным. В частности, не установлены оптимальные условия для наблюдения данного эффекта, а также неясен механизм дробного (полуцелого) квантования проводимости. В данной связи основная цель настоящей работы заключалась в изучении особенностей проявления квантовых эффектов в электронном транспорте мемристивных структур на основе PРX с медными контактами при их переключении из высокоомного (OFF) состояния в низкоомное $(\mathrm{ON})$ и обратно.

\section{2. Материалы и методы}

Были изучены мемристивные структуры конденсаторного типа. Слои РРX (толщиной $\sim 100$ нм) были нанесены на стеклянную подложку с нанесенным слоем ITO (оксид индия-олова), выступающим в качестве нижнего электрода, методом газофазной поверхностной полимеризации с использованием системы вакуумного осаждения SCS Labcoater PDS 2010. Димер парилена (2.2-парациклофан или его производные) превращается в газообразный мономер, который при осаждении полимеризуется на подложке при комнатной температуре. Промежуточная жидкая фаза или отдельный цикл отверждения отсутствуют. При используемых уровнях вакуума газообразный мономер одинаково осаждается на все стороны подложки, в результате чего получается равномерное покрытие.

Верхние металлические электроды представляли собой слои меди $(\mathrm{Cu})$ толщиной $\sim 500 \mathrm{HM}$, полученные ионным распылением через теневую маску. Размеры верхних электродов составляли $0.2 \times 0.5 \mathrm{Mм}$. Полученные мемристивные структуры $\mathrm{Cu} / \mathrm{PPX} / \mathrm{ITO}$ были изучены с использованием аналитической зондовой станции Cascade Microtech PM5. Электрофизические измерения проводились при комнатной температуре с помощью источника-измерителя Keithley 2636B.

\section{3. Результаты и обсуждение}

Квантование проводимости наблюдается в образцах $\mathrm{Cu} / \mathrm{PPX} / \mathrm{ITO}$ при измерении вольт-амперной характеристики $(\mathrm{BAX})$ с малой скоростью развертки по напряжению $(0.001 \mathrm{~B} / \mathrm{c})$ при переходе из низко- в высокоомное состояние и медленным изменением тока $\left(10^{-7} \mathrm{~A} / \mathrm{c}\right)$ при переходе из высоко- в низкоомное состояние. При этом выбор параметра развертки (напряжение или ток) определяется необходимостью возникновения отрицательной обратной связи при РП. Так, например, при РП из низков высокоомное состояние разрыв проводящего мостика и, как следствие, увеличение сопротивления структуры вызывают падение силы тока, протекающего через нее, при определенном уровне напряжения (в ходе развертки по напряжению). Это в свою очередь препятствует дальнейшей деструкции филамента, способствуя его стабилизации на определенном уровне проводимости. 


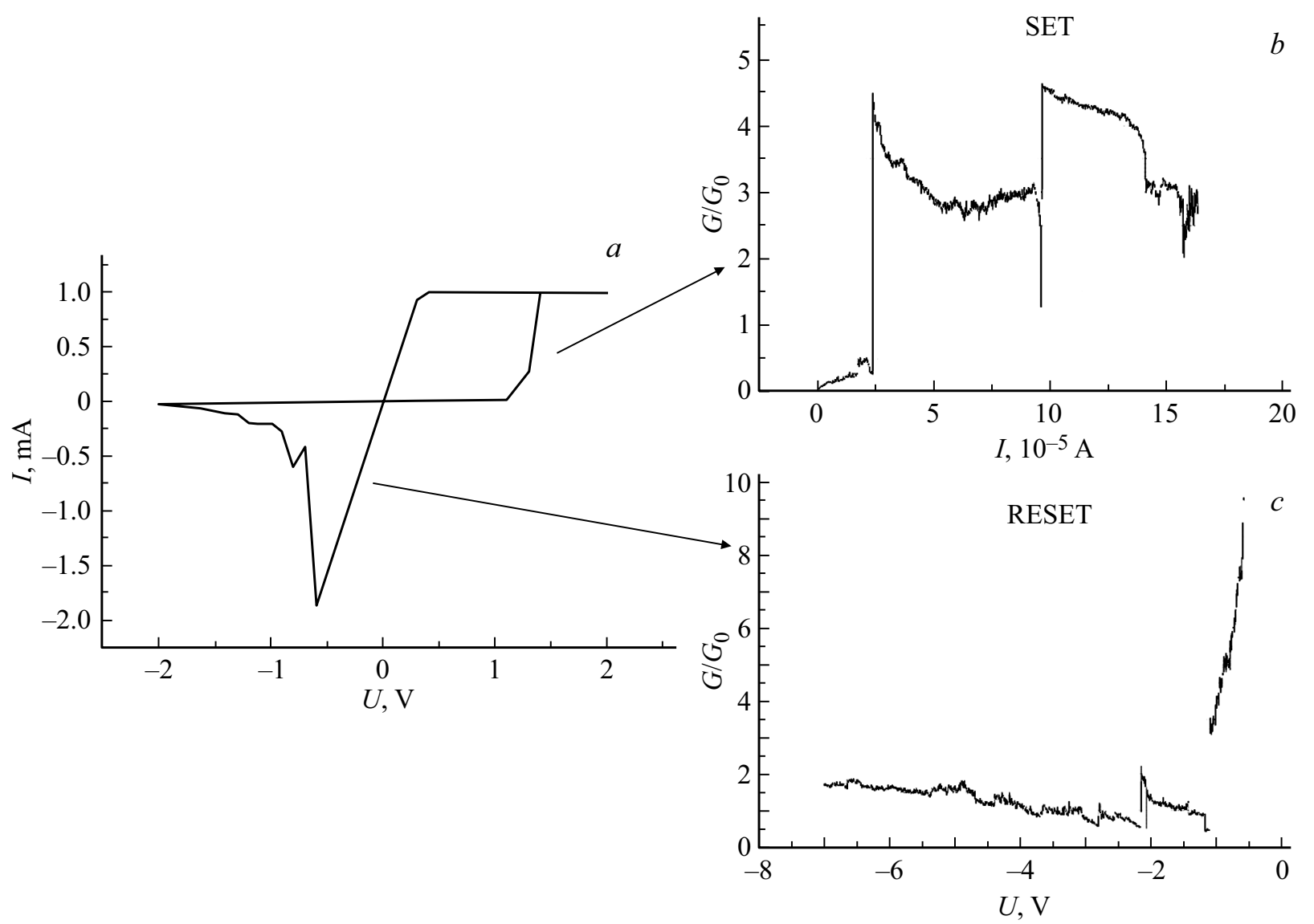

Pис. 1. Типичная BAX образцов $\mathrm{Cu} / \mathrm{PPX} / \mathrm{ITO}$ в режиме развертки по напряжению с ограничением тока $(a)$; переключение из OFF в ON состояние в режиме развертки по току $(b)$; переключение из ON в OFF состояние в режиме развертки по напряжению $(c)$.

Напротив, при РП из OFF в ON состояние, в случае развертки $\mathrm{BAX}$ по напряжению, последовательное уменьшение сопротивления вызывает скачкообразный рост силы тока, что приводит к лавинообразному росту проводящего мостика, не позволяющему наблюдать стабильные уровни квантованной проводимости.

На рис. 1, $a$ представлена типичная ВАХ образцов $\mathrm{Cu} / \mathrm{PPX} / \mathrm{ITO}$, гистерезисный характер которой определяется эффектом РП. Измерения ВАХ выполнены в режиме ограничения по току на уровне +1 и -100 мА с целью предотвращения перегрева мемристивной структуры, сопровождаемого ее деградацией. Цикл ВАХ измерялся путем приложения к структуре напряжения $U$ в следующей последовательности: линейное по времени увеличение $U$ от 0 до положительного напряжения $U+$, далее понижение $U$ до отрицательного напряжения $U-$ и затем повышение напряжения до 0. Напряжение изменялось с шагом $0.1 \mathrm{~B}$ при длительности каждого шага 100 мс. При уменьшении шага до 0.0001 В можно наблюдать переключение из ON в OFF состояние скачками (квантами проводимости) (рис. 1,c). Как было сказано выше, для обнаружения этого эффекта при обратном переключении используется режим развертки по току: до заданного значения ток изменяется от 0 до $1 \mathrm{~mA} \mathrm{c}$ шагом 10 нА (рис. $1, b)$.
В обоих случаях проводимость в единицах квантов проводимости вычислялась следующим образом: измерялись напряжение и ток, из которых было получено значение проводимости, которое делилось на квант проводимости. При этом при каждом шаге измерения ВАХ производилась запись полученных значений проводимости, совокупность которых для 10 последовательных экспериментов отображена в виде гистограммы на рис. 2 .

Как видно из рисунка, полуцелые значения квантов хорошо наблюдаются как при переключении в проводящее состояние (рис. 2, $a$ ), так и при переключении в непроводящее состояние (рис. $2, b$ ). Также заметно, что некоторые пики на гистограмме более выражены, т.е. данные уровни проводимости стабильнее. Если привести мемристивную структуру к заданному кванту проводимости, а затем производить измерение зависимости проводимости от времени на малых величинах напряжения, то можно понять, насколько стабильно заданное значение кванта (аналог характеристики retention - стабильности резистивного состояния). На рис. 3 представлены зависимости проводимости мемристивной структуры от времени, измеренные для различных уровней квантованной проводимости: от 0.5 до 7.5 с шагом 0.5 . Каждая зависимость измерялась 3 раза, после чего для каждой точки по времени выбиралось медианное зна- 

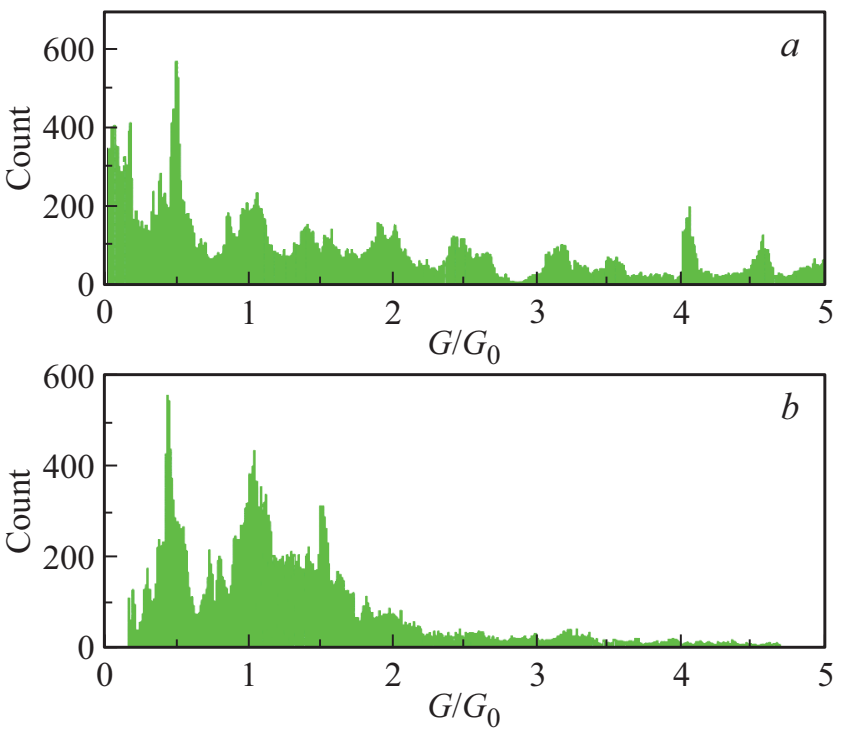

Рис. 2. Гистограмма совокупных значений проводимости при переключении мемристора $\mathrm{Cu} / \mathrm{PPX} / \mathrm{ITO}$ в низкоомное $(\mathrm{ON})$ состояние $(a)$ и при переключении в высокоомное (OFF) состояние $(b)$, полученных путем записи ВАХ с малой скоростью развертки по току и по напряжению соответственно. Данные по 10 последовательным измерениям для каждой гистограммы.

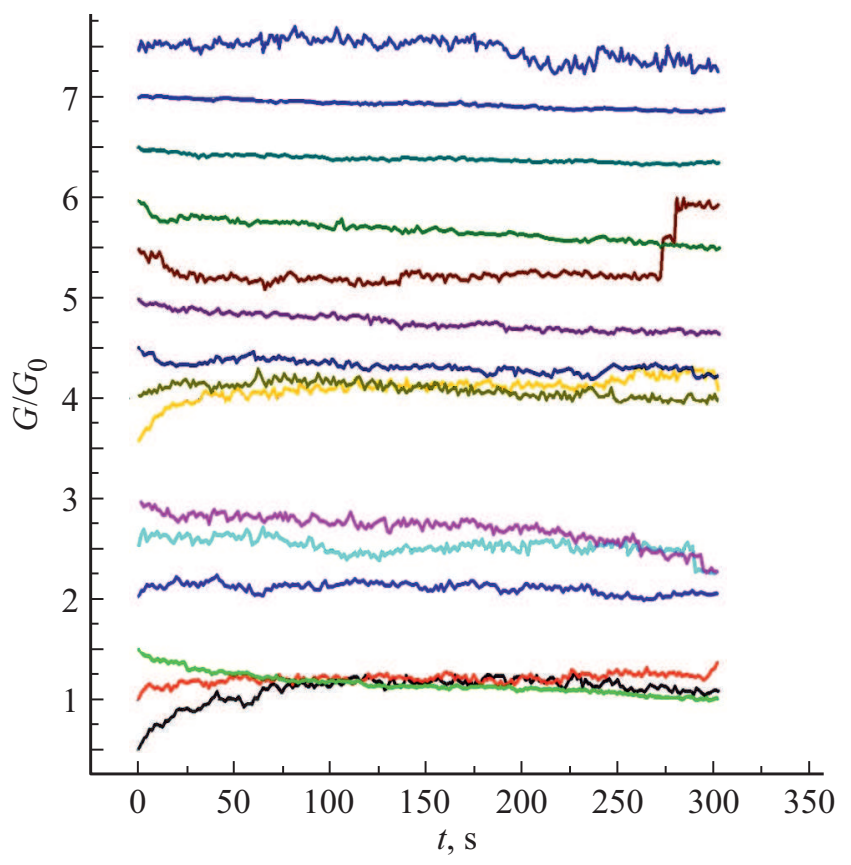

Рис. 3. Зависимости проводимости мемристивной структуры $\mathrm{Cu} / \mathrm{PPX} / \mathrm{ITO}$ от времени (медианные значения по 3 измерениям).

чение. Необходимое значение проводимости задавалось при помощи алгоритма с плавно подстраиваемой амплитудой [25]. Измерение сопротивления производилось 1 раз в секунду при напряжении чтения $0.1 \mathrm{~B}$.

На рисунке видно, что некоторые уровни квантованной проводимости действительно не являются стабиль- ными и в течение времени измерения (5 мин) стремятся перейти на другие уровни.

Квантование проводимости наблюдается в структурах $\mathrm{Cu} / \mathrm{PPX} / \mathrm{ITO}$, особенно при РП в низкоомное состояние, но механизм полуцелого квантования остается неясным. Также остается открытым вопрос, почему некоторые уровни проводимости (резистивные состояния) менее стабильны, что наблюдалось и ранее в экспериментах с этими структурами $[17,26]$. Относительно природы наблюдаемых полуцелых квантов проводимости в научном сообществе на сегодняшний день единой позиции нет. В некоторых работах сообщается, например, что такие состояния имеют место вследствие наличия адсорбированных примесей на металлических мостиках, которые и влияют на энергетическую структуру электронов [27]. Другое возможное объяснение - сильное взаимодействие между мостиками и водородным компонентом из окружающей их органической среды [24]. Если рассмотреть природу этого эффекта более глубоко, то можно предположить, что он может происходить из-за спонтанного снятия вырождения по спину энергетических уровней электронов вследствие эффекта Рашбы $[28,29]$. В целом анализ соответствующей литературы показывает, что вопрос о природе полуцелых состояний проводимости в таких системах является скорее спекулятивным, и основной физический механизм остается не выясненным. Для более детального анализа требуются дополнительные исследования.

\section{4. Заключение}

В работе изучено квантование проводимости при резистивном переключении мемристоров $\mathrm{Cu} / \mathrm{PPX} / \mathrm{ITO}$ при комнатной температуре. Были показаны методы измерения и продемонстрированы сходства и различия проявления эффекта квантования проводимости при переключении структур в высокоомное и низкоомное состояния. Также была продемонстрирована возможность задания стабильных квантовых состояний проводимости.

\section{Благодарности}

Работа выполнена при финансовой поддержке РФФИ (гранты 18-37-20014, 20-07-00696) на оборудовании Ресурсных центров при поддержке НИЦ „Курчатовский институт .

\section{Конфликт интересов}

Авторы заявляют, что у них нет конфликта интересов.

\section{Список литературы}

[1] D.B. Strukov, G.S. Snider, D.R. Stewart, R.S. Williams. Nature, 453, 80 (2008).

[2] M. Wojtyniak, K. Balin, J. Szade, K. Szot. Crystals, 10 (1), 33 (2020). 
[3] J.J. Yang, D.B. Strukov, D.R. Stewart. Nature Nanotechnol., 8, 13 (2013).

[4] Б.С. Швецов, А.В. Емельянов, А.А. Миннеханов, К.Э. Никируй, А.А. Несмелов, М.Н. Мартышов, В.В. Рыльков, В.А. Демин. Росс. нанотехнологии, 14 (1-2), 83 (2019).

[5] D. Ielmini. Semicond. Sci. Technol., 31, 063002 (2016).

[6] D. Ielmini, R. Waser (eds). From Fundamentals of Nanoionic Redox Processes to Memristive Device Applications (Weinheim, Wiley-VCH Verlag GmbH \& Co, 2016).

[7] A. Mehonic, A.L. Shluger, D. Gao, I. Valov, E. Miranda, D. Ielmini, A. Bricalli, E. Ambrosi, C. Li, J.J. Yang, Q. Xia, A.J. Kenyon. Adv. Mater., 30, 1801187 (2018).

[8] M. Lin, Q. Chen, Z. Wang, Y. Fang, J. Liu, Y. Yang, W. Wang, Y. Cai, R. Huang. Polymers, 9 (8), 310 (2017).

[9] W. Wang, M. Wang, E. Ambrosi, A. Bricalli, M. Laudato, Z. Sun, X. Chen, D. Ielmini. Nature Commun., 10, 81 (2019).

[10] E.R.W. van Doremaele, P. Gkoupidenis, Y.J. van de Burgt. Mater. Chem. C, 7, 12754 (2019).

[11] E.R.W. van Doremaele, P. Gkoupidenis, Y.J. van de Burgt. Mater. Chem. C, 7, 127540 (2019).

[12] J. Del Valle, J.G. Ramírez, M.J. Rozenberg, I.K. Schuller. J. Appl. Phys., 124, 211101 (2018).

[13] Y. Li, Z. Wang, R. Midya, Q. Xia, J.J. Yang. J. Phys. D: Appl. Phys., 51, 503002 (2018).

[14] Y. Cai, J. Tan, L. YeFan, M. Lin, R. Huang. Nanotechnology, 27 (27), 275206 (2016).

[15] Y. Van de Burgt, A. Melianas, S.T. Keene, G. Malliaras, A. Salleo. Nature Electronics, 394, 386 (2018).

[16] N.R. Hosseini, J.-S. Lee. Adv. Funct. Mater., 25, 5586 (2015).

[17] A.A. Minnekhanov, A.V. Emelyanov, D.A. Lapkin, K.E. Nikiruy, B.S. Shvetsov, A.A. Nesmelov, V.V. Rylkov, V.A. Demin, V.V. Erokhin. Sci. Rep., 9, 10800 (2019).

[18] Б.С. Швецов, А.Н. Мацукатова, А.А. Миннеханов, А.А. Несмелов, Б.В. Гончаров, Д.А. Лапкин, М.Н. Мартышов, П.А. Форш, В.В. Рыльков, В.А. Демин, А.В. Емельянов. Письма ЖТФ, 45 (21), 40 (2019).

[19] X. Zhu, W. Su, Y. Liu, B. Hu, L. Pan, W. Lu, J. Zhang, R.W. Li. Adv. Mater, 24, 3941 (2012).

[20] A. Mehonic, A. Vrajitoarea, S. Cueff, S. Hudziak, H. Howe, C. Labbe, R. Rizk, M. Pepper, A.J. Kenyon. Sci. Rep., 3, 2708 (2013).

[21] W. Yi, S.E. Savel'ev, G. Medeiros-Ribeiro, F. Miao, M.X. Zhang, J.J. Yang, A.M. Bratkovsky, R.S. Williams. Nature Commun., 7, 11142 (2016).

[22] K. Krishnan, M. Muruganathan, T. Tsuruoka, H. Mizuta, M. Aono. Adv. Funct. Mater, 27, 1605104 (2017).

[23] R. Landauer. IBM J. Res. Dev., 1, 223 (1957).

[24] W. Xue, S. Gao, J. Shang, X. Yi, G. Liu, R. Li. Adv. Electron. Mater., 2019, 1800854 (2019).

[25] К.Э. Никируй, А.В. Емельянов, В.А. Демин, В.В. Рыльков, А.В. Ситников, П.К. Кашкаров. Письма ЖТФ, 44 (10), 20 (2018).

[26] A.A. Minnekhanov, B.S. Shvetsov, M.M. Martyshov, K.E. Nikiruy, E.V. Kukueva, M.Yu. Presnyakov, P.A. Forsh, V.V. Rylkov, V.V. Erokhin, V.A. Demin, A.V. Emelyanov. Organic Electron., 74, 89 (2019).

[27] Y. Sun, D. Wen. J. Phys. Chem., 122, 10582 (2018).

[28] O. Goulko, F. Bauer, J. Heyder, J. von Delft. Phys. Rev. Lett., 113, 266402 (2014).

[29] R. Cuan, L. Diago-Cisneros. J. Appl. Phys., 110, 113705 (2011).

Редактор Г.А. Оганесян

\section{Conductance quantization in memristive structures based on poly-p-xylylene}

B.S. Shvetsov ${ }^{1,2}$, A.A. Minnekhanov ${ }^{2}$, A.A. Nesmelov ${ }^{2}$, M.N. Martyshov ${ }^{1}$, V.V. Rylkov ${ }^{2,3}$, V.A. Demin ${ }^{2}$, A.V. Emelyanov ${ }^{2,4}$

${ }^{1}$ Lomonosov Moscow State University

(Department of Physics),

119991 Moscow, Russia

${ }^{2}$ National Research Center Kurchatov Institute,

123182 Moscow, Russia

${ }^{3}$ Fryazino Branch of the Kotel'nikov Institute

of Radio Engineering and Electronics

of Russian Academy of Sciences,

141190 Fryazino Russia

${ }^{4}$ Moscow Institute of Physics and Technology, 141700 Dolgoprudny, Russia

Abstract The paper presents the results of a study at room temperature of the quantization effect of the conductivity of memristive structures based on the organic material poly-p-xylylene with resistive switching. Measurement methods are shown and a comparative analysis of the manifestation of the effect when switching structures to a high-resistance and low-resistance state is carried out. The possibility of setting stable quantum states of conductivity in memristive structures based on poly-p-xylylene is demonstrated. It is shown that some of these states have shortterm, and some long-term stability. The results obtained open up new possibilities for using the quantization effect of conductivity in the implementation of neuromorphic systems. 